\title{
Análisis de las dificultades que presentan los estudiantes universitarios en matemática básica
}

\section{Analysis of difficulties presented by university students in basic mathematics}

\author{
Roger Iván Soto Quiroz ${ }^{\mathrm{a} 1}$ y Daniel Noboru Yogui Takaesu ${ }^{2}$ \\ Universidad Peruana de Ciencias Aplicadas, Lima, Perú ${ }^{1}$ \\ Universidad Tecnológica del Perú , Lima, Perú \\ Orcid ID: https://orcid.org/0000-0002-3413-8425 1 \\ Orcid ID: https://orcid.org/0000-0002-6478-9093²
}

Recibido: 03 de julio de 2019

Aceptado 07 de octubre de 2019

\begin{abstract}
Resumen
El presente estudio pretende analizar las diferentes dificultades - tanto académicas como actitudinales - que se les presenta a los estudiantes universitarios que llevan por segunda vez el curso de Matemática Básica. Esta es una investigación cualitativa, con diseño fenomenológico, con aplicación de la técnica de la entrevista no estructurada. El sustento teórico se basa en la comprensión lectora, competencias escolares, aprendizaje significativo, razonamiento cuantitativo y las actitudes hacia la matemática. La muestra la conformaron 10 estudiantes que llevan por segunda vez el curso de Matemática Básica: 03 son de la carrera de Diseño Profesional Gráfico, 03 de Diseño de interiores y 04 de Diseño y Gestión de Modas; 03 docentes que enseñan el curso y 05 tutores académicos que realizan actividades de tutoría académica y talleres de reforzamiento del curso. Los resultados permitieron afirmar que se descubrieron seis dificultades: 1) Dificultad en la comprensión lectora de los problemas matemáticos, 2) dificultad en comprender contenidos matemáticos básicos, 3) dificultad con la asistencia a clases, 4) dificultad en el interés y motivación por el curso, 5) dificultad en repasar los temas matemáticos, 6) dificultad en la asistencia a tutoría y talleres de reforzamiento.
\end{abstract}

Palabras clave: comprensión lectora, aprendizaje significativo, actitudes hacia la matemática, habilidades matemáticas. 


\begin{abstract}
This article aims to analyze the different difficulties, both academic and attitudinal, that are presented in university students who take the Basic Mathematics course for second time. This work is a qualitative research, with phenomenological design, with application of the unstructured interview technique. The theoretical support is based on reading comprehension, school skills, meaningful learning, quantitative reasoning and attitudes towards mathematics. The sample was made up of 10 students who take the Basic Mathematics course for the second time: 03 of the Professional Graphic Design career, 03 of Interior Design and 04 of Fashion Design and Management; 03 teachers who teach the course and 05 academic tutors who carry out academic tutoring activities and workshops to reinforce the course. The results allowed us to affirm that they discovered six difficulties: 1) Difficulty in reading comprehension of mathematical problems, 2) difficulty in understanding basic mathematical contents, 3) difficulty with attending classes, 4) difficulty in interest and motivation for the course , 5) difficulty in reviewing mathematical topics, 6) difficulty in attending tutoring and reinforcement workshops.
\end{abstract}

Keywords: reading comprehension, meaningful learning, attitudes towards mathematics, mathematical skills.

\title{
Introducción
}

El curso de matemática ha ocasionado diversas reacciones en la mayoría de estudiantes de todos los niveles (inicial, primaria, secundaria y técnico-superior). Para algunos se convierte en un dolor de cabeza, generando trauma, temor, ansiedad; mientras que para otros refleja gozo o algo fácil de aprender. El gusto por la matemática y el desarrollo de sus habilidades se inician desde niños al contar, trazar, descubrir figuras, realizar operaciones elementales, resolver ecuaciones, expresarse en lenguaje simbólico, resolver problemas, etc.; pero también surgen las dificultades de su aprendizaje en aquellas personas que no la entienden, como ocurre con los estudiantes universitarios participantes del presente estudio.

En la universidad en estudio, en la asignatura de Matemática Básica, los estudiantes deben desarrollar habilidades de razonamiento cuantitativo como: interpretar, representar, calcular, analizar, etc. (Vergara, Fontalvo, Muñoz y Valbuena, 2015); y también habilidades más específicas como: extraer información de un texto matemático, identificar el valor de las variables necesarias para reemplazar en una fórmula, discriminar entre fórmulas, representar una proporción matemática, realizar operaciones básicas, resolver ecuaciones, entre otras. Investigaciones previas como la de Rojas (2018), han demostrado que existe un nivel alto de razonamiento cuantitativo en estudiantes de Ingeniería; asimismo, Ricra (2019) comprobó que el nivel de razonamiento cuantitativo de los estudiantes de Arquitectura fue suficiente con un 47,1\% y la dimensión "argumentación" presentó un nivel insuficiente con un 47,9\%. Por otro lado, los estudiantes de las carreras de Ingeniería y Arquitectura son afines a la matemática. En 
el caso de la presente investigación, está se dirige a estudiantes de la carrera de Diseño, que por lo general no muestran inclinación hacia la matemática.

Las dificultades que suelen presentarse en los estudiantes universitarios matriculados en Matemática Básica se exhiben a un nivel conceptual, procedimental, como a nivel actitudinal (Teoría de las competencias). En el nivel conceptual, las dificultades suelen estar relacionadas con aprender temas específicos como: tamaño de muestra, cambio de moneda, variaciones porcentuales, variables, entre otros; en el nivel procedimental, las mayores dificultades suelen ser que algunos estudiantes no comprenden el texto matemático, no identifican los datos adecuadamente, no infieren, no relacionan información anterior y posterior, no identifican el requerimiento del problema; en el nivel actitudinal, suelen presentar dificultades como: desinterés por el curso, poca participación y/o distracción en clase, o bien manipular sus celulares o conversando de otros temas con sus compañeros. Obviamente, eso afecta en sus calificaciones y desarrollo en la materia (Yao y Chiang, 2011; Bartual y Poblet, 2009; Delgado y Castillo, 2015; Gabalán y Vásquez, 2017). De esta forma, Mueller, Yankelewitz y Muher (2011) sostienen que, los estudiantes motivados en aprender matemática disfrutan cuando realizan sus tareas, comprenden y buscan el conocimiento.

En la universidad en estudio se observa poca o ninguna motivación hacia el curso de matemática, y los estudiantes carecen de recursos cognitivos para resolver problemas de la vida diaria (Rostaing, Bernuy, Borja, Martínez, Ayllón y Gómez, 2019). Asimismo, existen otras investigaciones cuyos resultados indican que existe una débil motivación intrínseca y extrínseca por parte de los estudiantes para el aprendizaje de la matemática, y también de los docentes para la enseñanza de la misma (Erazo, 2015; Navea, 2015; Jiménez, 2015, citados en Rostaing, Bernuy, Borja, Martínez, Ayllón y Gómez, 2019). Algunas investigaciones demuestran que, si el estudiante está desmotivado, su rendimiento académico disminuirá; además, al afrontar los problemas que tienen que resolver los estudiantes no toman en cuenta sus conocimientos previos; por lo tanto, los problemas no son significativos para ellos (Soto, 2018; Casis, Rico y Castro, 2017; Almonacid, Gutiérrez y Pullo, 2018).

En el presente estudio, se analizarán seis aspectos importantes que guardan relación con los estudiantes universitarios participantes que llevan por segunda vez el curso de Matemática básica. 


\section{Importancia de la comprensión lectora de los problemas matemáticos}

En los problemas matemáticos es importante que el estudiante descifre el sentido del texto y reconozca el contexto o situación del problema; por ejemplo, situaciones sobre “elecciones municipales", "día del shopping", "licenciamiento de universidades", “promociones y ofertas", entre otras (Viramonte, 2016). En la comprensión del texto, el estudiante debe identificar y relacionar toda la información de la situación problemática que se le brinda en forma verbal, mediante tablas, gráficos (de barras, circular, histograma, ojiva, polígono de frecuencias), en lenguaje simbólico (fórmulas, modelos matemáticos, ecuaciones), todas las cuales brindan información relevante para la solución de un problema.

\section{Importancia de los contenidos matemáticos básicos}

Existen contenidos básicos y habilidades matemáticas elementales que provocan complicación en los estudiantes. Estos ingresan a la universidad con dificultades que traen desde la escuela (porcentajes, operaciones con números reales, ecuaciones, funciones, interpretación de tablas y gráficos, entre otras). Al respecto, López (2013) manifiesta que, en la teoría de las competencias, los estudiantes desarrollan diferentes saberes: comprensión, interpretación, procesamiento de pensamiento, desempeño adecuado en el contexto del problema y comportamiento apropiado en diversos escenarios.

\section{Importancia de la asistencia a clases}

Los contenidos matemáticos son secuenciales, por lo general si un estudiante que es apto en matemática se pierde una clase, se le hace fácil nivelarse; mientras que para aquellos que tienen dificultades en la materia, les costará mucho su nivelación. Yao y Chiang (2011) encontraron que, estudiantes que faltaban a algunas clases-en especial a las primerastuvieron menor promedio en sus calificaciones en comparación con los que sí asistieron. Asimismo, Bartual y Poblet (2009) descubrieron que la asistencia continua a clases aumenta el porcentaje de aprobados de un curso. Igualmente, Delgado y Castillo (2015) demostraron que la dedicación de los estudiantes y su asistencia continua a clases producían incidencia positiva en sus calificaciones.

\section{Importancia del interés y motivación del estudiante por el curso}

Por lo general, los estudiantes universitarios cuyas carreras profesionales no son afines a la matemática sienten cierto rechazo, temor, desinterés y desmotivación hacia esa materia, y los estudiantes de la carrera de Diseño no son la excepción a ello. Que los estudiantes se interesen por la Matemática Básica va a depender del nivel de significatividad que provoque en 
ellos resolver los problemas: si estos son útiles o no para ellos, si despiertan o no su interés y curiosidad, si relacionan sus conocimientos previos con los recién adquiridos, etc. (Alcaraz, 2002; Guerrero, 2014; Roig, 2009). Asimismo, Cantoral, Covián, Farfán, Lezama y Romo (2008) sustentan que "El aprendizaje significativo se caracteriza porque tiene en cuenta los intereses, necesidades y realidades del alumno, despertando el interés en aprender lo que considera valioso (motivación)" (p.45).

\section{Importancia en repasar los temas matemáticos para afianzar los conocimientos}

Repasar los temas de matemática consiste básicamente en practicar los problemas que ya se han desarrollado en clase o los propuestos en las tareas, materiales de clase, libros, prácticas o exámenes, etc. Repasar los temas se puede realizar de forma individual, entre dos o más personas en grupos de estudio; también pueden ser con docentes, tutores, amigos o familiares. Para los estudiantes con dificultades en matemática, es importante la práctica continua de problemas matemáticos.

\section{Importancia de la asistencia a tutoría y talleres de reforzamiento para estudiantes que requieren asesoría académica}

La teoría del desarrollo cognitivo de Vygotsky tiene que ver con la forma en que el estudiante socializa con su entorno para aprender: los alumnos comparten sus clases en grupos de estudio, tutorías académicas, talleres de reforzamiento y en algunos casos con sus pares que repasan los temas con otras personas (Cantú, De Alejandro, García y Leal, 2017). Cabe señalar que la tutoría académica es una actividad que la universidad en estudio emplea como apoyo a los estudiantes, un espacio de aprendizaje personalizado, donde el estudiante se reúne con un tutor y resuelven todas las dudas matemáticas. Asimismo, los talleres de reforzamiento son actividades de repaso de los temas desarrollados en clase, de forma grupal, con diversos horarios para esta actividad.

Los estudiantes bajo el enfoque constructivista van construyendo día a día sus propios aprendizajes como consecuencia de la interacción de sus habilidades cognitivas, sociales, afectivas y de comportamiento, encontrándole significado a lo que producen. En este caso, el docente de matemática se constituye en un factor importante en la superación de dificultades que tienen los estudiantes para aprender matemática; por ello sus sesiones deben ser motivadoras, aplicando estrategias constructivas, promoviendo el aprendizaje significativo y tomando en cuenta los saberes previos de los estudiantes para que puedan conectarse a los nuevos (Cantú, De Alejandro, García y Leal, 2017). 
Por tanto, el presente estudio se realiza con el interés de explorar de forma cualitativa las principales dificultades que experimentan los estudiantes universitarios, con la finalidad de proponer (posterior al estudio) una estrategia de solución particular a cada dificultad presentada. En este sentido, el objetivo general del presente estudio fue analizar las dificultades por la que pasan los universitarios que llevan por segunda vez la asignatura de Matemática Básica.

\section{Método}

Este es un estudio con enfoque de investigación cualitativa, donde se exploran las principales dificultades que experimentan los participantes del estudio. A su vez, se orienta por un diseño fenomenológico, pues focalizó el análisis de las experiencias de los estudiantes que han desaprobado el curso de Matemática Básica, las razones para la desaprobación de la materia, sus errores académicos, deficiencias actitudinales y las reflexiones que han hecho para corregir sus equivocaciones, con el propósito de aprobar posteriormente el curso.

Para la investigación se empleó la técnica de la entrevista no estructurada. El estudio se realizó en una universidad privada cuya misión es la exigencia y la innovación, y se encuentra ubicada en la ciudad de Lima, Perú. Los estudiantes participantes pertenecen a la Escuela profesional de Diseño, donde se observa su escaso interés por la matemática.

En cuanto al procedimiento, se efectuaron los siguientes pasos:

Paso 1: Se trabajó con una muestra no probabilística y se seleccionó de modo intencional a los participantes del estudio - la muestra de conveniencia (Guzmán, Bastidas y Mendoza 2019). Esta muestra estuvo conformada por: 10 estudiantes matriculados por segunda vez en la asignatura de Matemática Básica de la Carrera Profesional de Diseño (04 de Diseño y Gestión de Modas, 03 de Diseño Profesional Gráfico y 03 de Diseño de Interiores), 03 docentes que les enseñan Matemática Básica y 05 tutores académicos, todos provenientes de una universidad privada de Lima, Perú.

Paso 2: Se elaboró una entrevista no estructurada, de manera individual, con una pregunta inicial para los estudiantes: “¿Qué dificultades tienes en el curso de Matemática Básica?” Asimismo, para los docentes la pregunta de partida fue “¿Qué dificultades para aprender el curso tienen sus estudiantes matriculados por segunda vez?" Mientras que, en el caso de los tutores, la pregunta fue “¿Qué dificultades observa en los estudiantes que llevan el curso por segunda vez y que asisten a la tutoría académica o a los talleres de reforzamiento?”

En el caso de los estudiantes, para una mejor direccionalidad y facilidad en la identificación de los contenidos temáticos que les parecieron complicados, se les indicó que porten su examen parcial y las prácticas calificadas del curso. Luego de realizar la pregunta 
inicial, se procedió a realizar las repreguntas sobre las subcategorías que mencionaban los participantes. Las entrevistas se realizaron en los ambientes de la universidad en estudio con el consentimiento del coordinador del curso y de todos los participantes.

Paso 3: Luego de realizar la primera pregunta a los participantes, se fueron anotando las subcategorías que surgieron en el transcurso de toda la entrevista. Algunas subcategorías fueron similares a las encontradas en la revisión bibliográfica, mientras que otras fueron nuevas subcategorías.

Paso 4: Se transcribieron las entrevistas, luego el análisis de la información permitió establecer seis subcategorías comunes a todos los participantes.

Paso 5: Se presentaron los resultados de la investigación. Se transcribieron, analizaron e interpretaron frases de los participantes que hacían referencia a cada subcategoría.

Paso 6: Se establecieron las conclusiones.

\section{Resultados}

Los resultados muestran seis dificultades (subcategorías) que manifestaron todos los participantes del estudio.

\section{Dificultad en la comprensión lectora de los problemas matemáticos}

Los problemas matemáticos que se desarrollan en las clases y se evalúan en los exámenes son de tipo caso, de una historia real o adaptada, con mucha información, donde el estudiante debe leer todo el documento en su totalidad, inclusive las referencias al pie de página. Para ello, los estudiantes deben aplicar algunas técnicas de comprensión de lectura como subrayar, resaltar, relacionar ideas, tener concentración, integrar el significado de lo que lee, lo que les permitirá resolver con éxito los problemas.

\section{Estudiante 1:}

"Los problemas son todos de comprensión lectora y eso es una de mis mayores dificultades. Son los problemas textuales que te ponen para que tú los comprendas, entonces tengo que pensar muy bien en lo que quiere decir el fragmento del problema y después siempre tengo que estar conectada con la anterior pregunta."

\section{Estudiante 2:}

"Del texto, tengo que leer todo, resalto lo más importante, leo las referencias del pie de página puesto que a veces hay información valiosa para resolver el problema. Tengo que concentrarme mucho para entender el texto."

Los estudiantes deben leer todo el texto del problema y relacionar los datos, algunas veces la información se encuentra de forma textual, gráfica, tabular o simbólica. 
Docente 1:

"Tienen dificultades de comprensión lectora para resolver los problemas. Deben relacionar toda la información del texto, ellos dicen: yo he jalado el curso porque no he leído bien."

Tutor 1:

"Tienen una dificultad en la comprensión lectora del problema matemático, la información la tienen en el texto, pero se les complica identificar la información sustancial."

\section{Dificultad en comprender contenidos matemáticos básicos}

Entre los contenidos temáticos que tienen dificultad se tiene: cambio de moneda, variaciones porcentuales, valor $\mathrm{z}$, redondeo y variables.

\section{Estudiante 1:}

"No entiendo la compra y venta de dólares. Las variaciones porcentuales cuando aparecen en gráficos."

\section{Estudiante 6:}

"Completar la tabla de distribución de frecuencias de variable cuantitativa continua."

\section{Docente 2:}

"Se les complica encontrar el valor z para encontrar el tamaño de muestra, piensan que el subíndice se multiplica, también se complican en el tema de variaciones porcentuales."

\section{Tutor 1:}

"Tienen muchas dificultades con la variación porcentual; por ejemplo, cuando se les presenta un gráfico de variación porcentual, es bien complicado para ellos el reconocimiento de nombre de variables de estudio y los tipos de variable, una vez un alumno me dijo que variable es $40 \%$, otro mencionó variable 'hombres' cuando en realidad la variable es "sexo."”

Tutor 2:

"Encontrar tamaño de muestra (por ejemplo, el subíndice del valor z, el valor p y q, el redondeo por exceso, se olvidan bastante). Se les complica mucho los temas de matemática y lo asocian con algunas malas experiencias en el colegio, con situaciones de baja autoestima o actitudes negativas hacia la matemática."

\section{Estudiante 2:}

"Se me complica el tema de variación porcentual en tablas y gráficos, eso de identificar el valor presente y el valor pasado, reemplazarlo en la fórmula. En un examen vino una pregunta de estas y no la hice. Desde el colegio no sé nada de matemática, se me complica mucho."

\section{Estudiante 3:}

"Tengo dificultades con problemas de tipo de cambio de moneda, redondear un número no sé si es al menor entero o al mayor entero. He sido muy mala en matemática desde la escuela." 


\section{Docente 3:}

"Una vez un estudiante dijo: no sirvo para la matemática, nunca me ha gustado, siempre he sido malo en ello. Pero, en el primer examen (control 1) lo aprobé con 14, porque si lo hubiese desaprobado, ya me hubiese retirado del curso. No sabía ni colocar la línea de división, ni qué operación se resuelve primero. Me ayudó el ser ordenado, y con esfuerzo, practicando en casa, asistiendo a las tutorías, he podido enfrentar con éxito, aprobar y aprender la matemática. He sacado 18 de promedio en el curso.”

\section{Dificultad con la asistencia a clases}

Generalmente la inasistencia o tardanza a las clases es de índole actitudinal, un descuido de su parte, lo que conlleva a la perdida de la secuencia del tema y si llegan tarde encuentran el desarrollo del problema a medias y esto afecta a sus calificaciones.

\section{Estudiante 1:}

"Si no vine a clases un día y no asistí a tutoría en esa semana ese sí que es un gran problema para poder entender el tema y los temas que siguen, ese error lo cometí el ciclo pasado ahora no."

\section{Estudiante 5:}

"Llego tarde a las clases de matemática, generalmente 20 minutos tarde. Llegar temprano a clases también es importante, puesto que si llegas 20 minutos tarde ya te perdiste toda la introducción del tema, en mi caso eso sí es una gran dificultad mientras que para otros que son más hábiles en el curso es muy fácil se pone al día rápidamente.”

\section{Docente 1:}

"Se descuidan mucho, tanto así que faltan a clases, en el aula te quedan mirando, no agarran el lapicero para desarrollar el material de clase, no toman apuntes, algunos solo toman fotos."

\section{Dificultad en el interés y motivación por el curso}

El desinterés y la falta de motivación de los participantes se manifiesta en sus distracciones con el celular, conversaciones de temas ajenos a lo que se desarrolla en la clase o por confiarse demasiado.

\section{Estudiante 2:}

"Me distraigo mucho con el celular en clase, por ello, lo apago para prestar atención a los temas que hace el profesor."

\section{Estudiante 3:}

"Me distraía mucho con el celular en plena clase, usaba el WhatsApp, Facebook, ahora también me tienta, pero me controlo." 


\section{Tutor 1:}

"Los alumnos mencionan que han desaprobado el curso por dejadez de su parte, se han confiado, han pensado que el curso iba a ser sencillo aprobarlo, no han comprendido que aprobarlo es un proceso."

Por lo general, estas dificultades se presentaban más en el ciclo pasado, mientras que en el presente ciclo han tomado conciencia de sus errores y se han propuesto mejorar.

\section{Estudiante 4:}

"El ciclo pasado, me distraía muchísimo en clase de matemática, tenía un grupo de amigos de mi carrera y me distraían porque conversaban mucho o hacían mucha chacota en clase o hacía reír a mis amigos o salíamos de clase y después volvíamos. Pero ahora no, es totalmente distinto ya no estoy con mi grupo de amigos de antes, ahora ya no me distraigo, presto atención totalmente, me ubico adelante antes me ubicaba atrás y hacía cualquier cosa menos prestar atención, me distraía con el celular porque prefería eso a atender la clase del profesor. Ahora, presto atención y entiendo las explicaciones del profesor."

El desinterés en el curso también se debe a la percepción de los participantes de que el curso no tiene utilidad práctica en su carrera profesional de Diseño.

\section{Estudiante 5:}

"Mi carrera es diseño gráfico, yo ya estoy trabajando en mi carrera y la verdad que aplico poco o casi nada de matemática en lo que hago, siento que a veces no tiene sentido el curso."

\section{Docente 2:}

"En los alumnos de Diseño, no veo el interés por aprender el curso, lo que veo es que solo quieren aprobar el curso y punto. Me dicen que eso no lo aplicarán en su carrera."

\section{Dificultad en repasar los temas matemáticos}

Las actividades de extensión, la transferencia de los aprendizajes y la preparación para los exámenes permiten que las habilidades matemáticas se asimilen, interioricen y se desarrollen. Los estudiantes participantes pertenecen a una carrera donde a la mayoría no les gusta el curso, han tenido traumas o miedo a la matemática, por ello es importante que practiquen los temas fuera del aula.

\section{Estudiante 2:}

"Solo cuando hay examen, foros o controles me pongo a repasar los temas, en otros momentos libres que tengo ni veo los temas. El ciclo pasado no entendía el curso, llegué al examen parcial y no resolví nada del examen porque no sabía nada, ahora es diferente porque estoy poniendo más esfuerzo en estudiar los temas. Ahora tomó apuntes que me sirven para repasar y entender los temas." 
Superar esta dificultad implica la aplicación de técnicas de estudio como la organización de un horario de estudio en casa, búsqueda de información, repetición de la resolución del problema, estudio en grupo, entre otras.

\section{Estudiante 3:}

"Ahora estudio en mi casa por las noches, le dedico mínimo dos horas y máximo tres horas a repasar los temas de matemática, dos o tres veces por semana. Repaso las diapositivas del aula virtual, y si tengo algunas dudas, busco información en internet para tener una guía. De lo contrario, le pregunto a alguien que también sabe matemática para que me oriente y voy practicando, porque creo que así he podido mejorar, estaba peor en el ciclo pasado como no practicaba entonces desaprobé el curso. Sacaba mala nota porque no practicaba los temas, no repasaba nada en casa, no le dedicaba tiempo al curso."

Todos los participantes ya conocen los temas y por ello algunos piensan que ya no necesitan repasarlos.

\section{Docente 1:}

"Prevalece en ellos el conformismo, piensan que ahora si van a pasar el curso, algunos se sienten tan cómodos que dicen que ya no van a estudiar mucho, que ya han llevado el curso y saben cómo manejarlo."

\section{Dificultad en la asistencia a tutorías y talleres de reforzamiento}

Para los estudiantes participantes, las tutorías académicas y los talleres de reforzamiento que desarrollan los tutores antes no tenían tanta importancia como ahora. Los que no pueden asistir a estas actividades buscan otras alternativas como buscar ayuda con amigos y/o familiares que saben de matemática.

\section{Estudiante 7:}

"No asisto ni a tutorías ni a talleres, tengo una amiga de un ciclo superior que me ayuda, me explica todo y fácil entiendo."

\section{Estudiante 8:}

"El ciclo pasado no asistía, pero ahora asisto a tutorías de 2 a 4 horas a la semana para repasar con el tutor los temas desarrollados en la semana de clases, poder entender los problemas y lograr por fin aprobar este curso que llevo por segunda vez."

\section{Estudiante 9:}

"He asistido a talleres de reforzamiento y además la tutora me explicaba brevemente muy bien los temas que no entendía. En este ciclo no asisto a tutorías ni talleres, en casa suelo estudiar con mi hermana, ella sabe de matemática y me ayuda mucho con los temas que no entiendo."

Para algunos participantes, existe cruce de sus horarios de clase con los de tutoría, por ello, su inasistencia a estas actividades. 


\section{Estudiante 10:}

"Una vez asistí a tutoría académica y me fue bien, tenía bastante paciencia, considero que ayuda mucho el asistir a tutoría, pero lo malo es el horario que existe, se me cruzan con las clases que tengo."

Los participantes que asisten a la tutoría presentan buena actitud, desean entender los temas y se han propuesto aprobar el curso.

\section{Estudiante 8:}

"Gracias a las tutorías he aprendido bastante, los tutores tienen mucha paciencia, buen trato y generan confianza.”

\section{Tutor 3:}

"Suelen venir los alumnos interesados en el servicio, algunos quieren que se les explique toda la clase que faltó, otros desean que se les absuelva todas sus dudas, quieren aprender más, profundizar el tema."

Tutor 4:

"Asisten más a las tutorías en fechas próximas a los exámenes."

\section{Tutor 5:}

"Asistía un alumno 'bica' (que lleva el curso por segunda vez) que sacó buenas notas en todos sus exámenes, es de los que tienes que estar al pendiente de él."

\section{Tutor 1:}

“Tienen una situación de revancha, mayormente los alumnos 'bicas' que vienen a asesorías piensan que, si ya se equivocaron el ciclo pasado, ahora ya no les volverá a pasar. Los estudiantes que llevan el curso por segunda vez vienen a la tutoría con buena actitud, reservan la tutoría para dos horas, y si estoy libre se siguen quedando una hora más, traen sus materiales actuales y materiales pasados para poder reforzarlos, siendo su principal motivación obviamente aprobar el curso."

\section{Discusión}

Referente a la dificultad que presentan los estudiantes en la comprensión de lectura de los problemas matemáticos, se descubrió que los estos tienen complicaciones en leer todo el texto y relacionar las diversas formas en que se presenta la información en tablas, gráficos, símbolos, y textos verbales. La identificación de datos, la interpretación y la representación son habilidades importantes en esta etapa. Vernucci, Canet, Andrés y Burin (2017) demostraron que las habilidades de comprensión lectora y cálculo matemático son importantes para desempeñarse exitosamente en el colegio, en la universidad, en cualquier situación que se presenta en la vida cotidiana y para el aprendizaje en general.

La segunda dificultad manifestada en los estudiantes fue comprender los contenidos matemáticos básicos, como la regla de tres simple al realizar cambio de moneda o al encontrar 
la variación porcentual de dos cantidades; otro contenido elemental son las operaciones con números reales al calcular el tamaño de una muestra y realizar las aproximaciones. Estas dificultades se exteriorizan a través del decaimiento de su autoestima, miedo, pánico, y estrés por la matemática. Mato y De la Torre (2010) encontraron que los docentes influyen en la formación de actitudes (negativas o positivas) hacia la matemática y, por ende, en la motivación hacia su estudio.

La tercera dificultad descubierta en los estudiantes fue la inasistencia y tardanza a las clases, siendo esta básicamente un asunto actitudinal del alumno, lo cual trae consecuencias negativas que afectan su aprendizaje y, por ende, sus calificaciones. Las investigaciones indican que los estudiantes que suelen faltar a clases, por lo general, suelen tener bajas calificaciones (Yao y Chiang, 2011; Bartual y Poblet, 2009; Delgado y Castillo, 2015; Gabalán y Vásquez, 2017).

La cuarta dificultad referente al interés y motivación por el curso también es de índole actitudinal por parte de los estudiantes, pero está en los docentes y tutores desarrollar las clases amenas y que los problemas que propongan despierten el interés de sus alumnos. Casis, Rico y Castro (2017) encontraron en su investigación que, entre las tres categorías actitudinales (motivación, autoconfianza y ansiedad), la motivación del estudiante hacia la matemática tiene más orientación positiva. Asimismo, Elisondo, Donolo y Rinaudo (2009) sugieren que los docentes universitarios deben proponer actividades basadas en una situación concreta de resolución de problemas específicos aplicada a la vida real o a su carrera profesional.

La quinta dificultad referente a repasar los temas matemáticos, tiene que ver con una parte actitudinal y otra cognitiva. En la parte actitudinal, los estudiantes no organizan su tiempo para repasar los temas en casa solos o con las redes de apoyo: amigos, familiares, profesor particular, entre otros. En la parte cognitiva, como no entienden los temas, simplemente no los repasan, ni tienen técnicas de estudio adecuadas para practicar. Almonacid, Gutiérrez y Pullo (2017) encontraron que existe una falta de motivación e interés por repasar las resoluciones de problemas matemáticos.

La sexta dificultad encontrada es la falta de asistencia a las tutorías y talleres de reforzamiento que se brindan de forma gratuita a los estudiantes, pero que no aprovechan -de manera estratégica- el servicio brindado, algunos suelen asistir únicamente cuando se aproximan los exámenes. La investigación realizada por Zaldívar, Nava y Lizárraga (2018) demostró que la tutoría en matemática ayuda a mejorar el rendimiento académico de los estudiantes, sus calificaciones se elevan. 


\section{Conclusiones}

Se descubrieron seis dificultades que presentan los estudiantes que llevan por segunda vez el curso de matemática Básica, estas son: 1) Dificultad en la comprensión lectora de los problemas matemáticos, 2) dificultad en comprender contenidos matemáticos básicos, 3) dificultad con la asistencia a clases, 4) dificultad en el interés y motivación por el curso, 5) dificultad en repasar los temas matemáticos, 6) dificultad en la asistencia a tutoría y talleres de reforzamiento.

Se descubrieron otras dificultades, pero solo fueron mencionadas por algunos participantes, tales como: presencia de nervios frente a los exámenes, olvido de los temas, miedo frente a los exámenes, no utilizar adecuadamente la calculadora, no darle importancia al curso por ser una asignatura general pero sí a los cursos de especialidad, falta de adecuación a la vida universitaria; no se acomodan aún al aprendizaje por competencias y consideran que las actividades son las mismas que el ciclo pasado, aunque en este nuevo hay otras actividades.

En resumen, la mayoría de los estudiantes participantes han reflexionado sobre sus actitudes negativas, los errores que cometieron el ciclo pasado, las distracciones que tuvieron antes, pero ahora dan muestras de un cambio de actitud, se han propuesto superar estas dificultades, dedicándose más a sus tareas universitarias y su meta es aprobar el curso.

\section{Referencias}

Alcaraz, F. (2002). Didáctica y currículo: un enfoque constructivista. España: Universidad de Castilla-La Mancha.

Almonacid, M., Gutiérrez, L. y Pullo, N. (2017). La motivación y el aprendizaje en el área de matemática (Tesis de licenciatura). Universidad Nacional de Educación Enrique Guzmán y Valle, Lima, Perú.

Bartual, T. y Poblet, M. (2009). Determinantes del rendimiento académico en estudiantes universitarios de primer año de economía. Revista de Formación e Innovación Educativa Universitaria, 2(3), 172-181.

Cantoral, R., Covián, O., Farfán, R., Lezama, J. y Romo, A. (2008). Investigaciones sobre enseñanza y aprendizaje de las matemáticas: un reporte iberoamericano. España: Ediciones Díaz De Santos.

Cantú, D., De Alejandro, C., García, J. y Leal, R. (2017). Comprensión lectora, educación y lenguaje. Estados Unidos: Polibrio. 
Casis, M., Rico, N. y Castro, E. (2017). Motivación, autoconfianza y ansiedad como descriptores de la actitud hacia las matemáticas de los futuros profesores de educación básica de Chile. PNA, 11(3), 181-203.

Delgado, M. y Castrillo, L. (2015). Efectividad del aprendizaje cooperativo en contabilidad: una contrastación empírica. Revista de Contabilidad, 18(2), 138-147.

Elisondo, R., Donolo, D. y Rinaudo, M. (2009). Ocasiones para la creatividad en contextos de educación superior. Revista de Docencia Universitaria. Recuperado de http://www.um.es/ead/Red_U/4/elisondo.pdf.

Gabalán, J. y Vásquez, F. (2017). Rendimiento académico universitario y asistencia a clases: Una visión. Revista Educación, 41(2). doi: http://dx.doi.org/10.15517/revedu.v41i2.18477

Guerrero, M. (2014). Metodologías activas y aprendizaje por descubrimiento. Las TIC y la Educación. Marpadal Interactive Media S.L.

Guzmán, K., Bastidas, B. y Mendoza, M. (2019). Estudio del rol de los padres de familia en la vida emocional de los hijos. Revista de investigación Apuntes Universitarios, 9(2), 6172.

López, M. (2013). Aprendizaje, competencias y TIC. México: Pearson.

Mato, M. y De la Torre, E. (2010). Evaluación de las actitudes hacia las matemáticas y el rendimiento académico. PNA, 5(1), 197-208.

Mueller, M., Yankelewitz, D. y Maher, C. (2011). Sense making as motivation in doing mathematics: Results from two studies. The Mathematics Educator, 20(2), 33-43.

Roig, V. (2009). Nuevas tecnologías de enseñanza-aprendizaje en la universidad. España: Ediciones Universidad de Salamanca.

Ricra, J. (2019). El aprendizaje cooperativo y la competencia razonamiento cuantitativo en estudiantes de matemática del primer ciclo de una Universidad Privada (Tesis de maestría). Universidad de San Martín de Porres, Lima, Perú.

Rojas, J. (2018). Razonamiento cuantitativo y la investigación formativa en estudiantes de ingeniería de la Universidad Peruana de Ciencias Aplicadas, 2018 (Tesis de maestría). Universidad de San Martín de Porres, Lima, Perú.

Rostaing, G., Bernuy, L., Borja, C., Martínez, E., Ayllón, M. y Gómez, I. (2019). Motivación de logro y resolución de problemas con funciones lineales en una universidad de Surco. Revista de investigación Apuntes Universitarios, 9(1), 85-101. 
Soto, R. (2018). Principios que consideran los catedráticos al elaborar problemas matemáticos. Revista UCV-Scientia, 10(2), 132-137. doi: dx.doi.org/10.18050/RevUcvScientia.v10n2a2

Vergara, J., Fontalvo, J., Muñoz, A. y Valbuena, S. (2015). Estrategia didáctica para el fortalecimiento del razonamiento cuantitativo mediante el uso de las TIC. Matua, Revista del Programa de Matemáticas, 2 (2), 71-80. Universidad del Atlántico, Barranquilla, Colombia.

Vernucci, S., Canet, L., Andrés, M. y Burin, D. (2017). Comprensión Lectora y Cálculo Matemático: El Rol de la Memoria de Trabajo en Niños de Edad Escolar. Psykhe, 26(2), 1-13. doi: https://dx.doi.org/10.7764/psykhe.26.2.1047

Viramonte, M. (2015). Comprensión lectora: dificultades estratégicas en resolución de preguntas inferenciales. Buenos Aires: Ediciones Colihue.

Yao, J. y Chiang, T. (2011). Correlación entre la asistencia a clases y las calificaciones. Journal of Computing Sciences in Colleges, 27(2), 142-147.

Zaldívar, A., Nava, L. y Lizárraga, J. (2018). Influencia de la tutoría en el aprendizaje de matemáticas. Perspectiva del estudiante. RIDE. Revista Iberoamericana para la Investigación y el Desarrollo Educativo, 8(16), 493-515. doi: https://dx.doi.org/10.23913/ride.v8i16.355 